\title{
Molecular genetic analysis of alloplasmic recombinant lines (Triticum dicoccum) - Triticum aestivum
}

\author{
Andrey Borisovich Shcherban \\ Laboratory of Molecular Genetics and \\ Cytogenetics of Plants, \\ Kurchatov Genomics Center, \\ ICG SB RAS Novosibirsk, Russia \\ atos@bionet.nsc.ru
}

\author{
Roman Nikolaevich Perfil'ev \\ Novosibirsk State Agrarian University \\ Novosibirsk, Russia \\ pervf.1999@gmail.com
}

\author{
Elena Artemovna Salina \\ Laboratory of Molecular Genetics and \\ Cytogenetics of Plants, \\ Kurchatov Genomics Center, \\ ICG SB RAS \\ Novosibirsk, Russia \\ sunday01@mail.ru
}

\begin{abstract}
Molecular markers were used to analyze the mitochondrial genome of the alloplasmic lines of wheat containing the nuclear genome of the hexaploid wheat $T$. aestivum against the background of the cytoplasm of tetraploid wheat T.dicoccum. Eight lines showed patterns of PCR and CAPS markers corresponding to the species $T$. aestivum, indicating the substitution of the mitochondrial genome of $T$. dicoccum during backcrossing with hexaploid wheat. In the D$\mathrm{N}-05$ line, the rps19 and orf256 gene markers corresponded to the parental species T.dicoccum. This line is promising in terms of studying the mechanisms of fertility restoration in hybrids with CMS. Also, using molecular marker, the nuclear gene Dreb-1, which is a regulator of drought tolerance, was analyzed and it was shown that line D-41-05 had an introgression of this gene from the B genome of $T$. dicoccum, which may lead to the previously established increased drought tolerance of this line.
\end{abstract}

Keywords - alloplasmic lines, common wheat, mitochondrial genome, drought resistance, gene

\section{Introduction}

In connection with the task of increasing the genetic diversity of the main cereal crop, common wheat, T. aestivum L. $(2 \mathrm{n}=42$; BBAADD $)$, alloplasmic lines, in which the nuclear genome of this species combines with the cytoplasm of an alien species as a result of remote hybridization and subsequent backcrossing with common wheat, are of great interest. Despite backcrossing, the nuclear genome of such lines may contain multiple introgressions of the maternal genome resulting from recombination. This may be important for breeding, in particular, for the selection of forms with high resistance to stress factors, both biotic and abiotic [1]. Nuclear cytoplasmic interactions, in particular, the influence of the mitochondrial genome, also make a great impact to the phenotypic variability, fertility, and viability of hybrids [2]. The modes of variability of both the nuclear and cytoplasmic genomes in the process of remote hybridization are still poorly understood.

Earlier, fertile alloplasmic wheat lines were obtained at the Institute of Plant Biology and Biotechnology of Kazakhstan. The results of preliminary experiments showed the resistance of some lines to increased salt concentration, as well as to water deficiency [3]. This work is aimed at analyzing the peculiarities in the organization of the mitochondrial genome of these lines, as well as the search for genes associated with various manifestations of drought tolerance, and the study of their organization and expression.

\section{Materials and Methods}

As a material we used 9 fertile alloplasmic wheat lines obtained from crossing () $T$. dicoccum Schrank $(2 \mathrm{n}=28$; BBAA) x (ふึ) T. aestivum L. cv. Mironovskaya 808. Total
DNA was isolated from 7-day old seedlings according to standard technique with sodium bisulfite.

\section{Mitochondrial genome analysis}

PCR markers of the following genes were taken for the analysis of mtDNA of alloplasmic lines: 1) orf256 - chimeric reading frame near the cytochrome oxidase gene (associated with cytoplasmic male sterility (CMS) [4]; 2) nad6mitochondrial gene encoding NADH-ubiquinone oxidoreductase (subunit 6); 3) rps19-p-pseudogene encoding a ribosomal protein [5]. PCR products were separated on a $2 \%$ agarose gel with the addition of ethidium bromide. The gel was photographed using GelDoc XR (BioRad, England). The orf 256 PCR product was excised from the gel, purified using a kit (QIAGEN, Germany) and sequenced using a bigdye terminator v3.1 cycle sequencing kit (Applied Biosystems, USA). Sequencing products were analyzed at the Collective Use Center "Genomika" of the SB RAS. Additionally, in the case of orf256, a CAPS marker was used, namely: the PCR product was digested with $T a q$ I restriction endonuclease (Sibenzyme, Novosibirsk) followed by electrophoresis on a $2 \%$ agarose gel.

\section{Analysis of the drought resistance regulator gene Dreb-1}

In this work, the marker of the Dreb- 1 gene described in [6] was used. PCR products were analyzed on a $2 \%$ agarose gel. To confirm the single-nucleotide substitution specific for the $T$. dicoccum Dreb-B1 gene, PCR products were digested with restriction endonuclease $B s t$ F5I (Sibenzyme) followed by electrophoresis in agarose gel. A $635 \mathrm{bp}$ fragment specific for $T$. dicoccum was excised from the gel and sequenced as described above.

\section{Results and Discussion}

Analysis of the mitochondrial genome of 9 alloplasmic wheat lines using molecular markers for the 3 above genes showed that 8 lines predominantly inherit the type of male parent mtDNA. These lines showed the patterns of PCR products and a CAPS marker identical to those of T. aestivum (Mironovskaya 808). Consequently, the restoration of fertility of alloplasmic recombinant lines (T. dicoccum) $-T$. aestivum correlates with the substitution of DNA sequences targeting $T$. dicoccum mtDNA. An exception is the D-N-05 line, in which the patterns of PCR markers for the rps 19 and orf 256 genes corresponded to the parental species T.dicoccum. Interestingly, the orf256 sequence in this line has $100 \%$ homology with the analogous sequence of the CMS line of $T$. timopheevii () x T. aestivum (X56186), however, unlike the latter, the D-N-05 line is fertile. In the future, we plan to identify genes of fertility restoration within the nuclear genome of the D-N-05 line.

As previously shown, the greatest drought tolerance is characteristic of the lines D-d-05b, D-b-05, D-41-05 [3]. The 
Dreb (Dehydration responsive element binding) family of genes are related to the very important regulatory genes that affect the tolerance to different abiotic stress factors including drought [7]. Using markers for one of the genes of this family, Dreb-1, we showed that in the nuclear genome of the droughttolerant line D-41-05 there is a copy of the Dreb-B1 gene (Fig. $1)$, which has a single nucleotide substitution characteristic of the sequence of Dreb-B1 T. durum [6], the tetraploid wheat species closest to $T$. dicoccum. Thus, it can be assumed that the increased drought tolerance of the D-41-05 line is associated with introgression of the $T$. dicoccum gene Dreb$B 1$ in the genome of common wheat. In the future, we plan to analyze the expression of Dreb-B1 under the influence of drought in alloplasmic lines differing by drought resistance.

\section{ACKNOWLEDGMENT}

The study was supported by the Kurchatov's Genomic Center (grant № 075-15-2019-1662).

\section{REFERENCES}

[1] V.P. Kholodova, T.S. Bormotova, O.G. Semenov, G. A. Dmitrieva, V. Kuznetsov, "Physiological mechanisms of adaptation of alloplasmic wheat hybrids to soil drought" Russ. J Plant Physiol., vol.54, pp. 480486, 2007.

[2] K. L. Liberatore, S. Dukowic-schulze, M. E. Miller, C. Chen, S.F. Kianian, "The role of mitochondria in plant development and stress tolerance" Free Radic. Biol. Med., vol. 100, pp. 238-256, 2016.

[3] N. V. Terletskaya, N. A. Khailenko, A. B. Iskakova, "Features of the reaction of alloplasmatic wheat lines seedlings on the effects of osmotic and salt stress" Vestnik Samarskogo Gosudarstvennogo Universiteta. Estestvenno-Nauchnaya Seriya, Issue 2(83), pp. 244-249, 2011.

[4] A.M. El-Shehawi, A.I. Fahmi, S.M. Sayed, M.M. Elseehy, "Genetic Fingerprinting of Wheat and Its Progenitors by Mitochondrial Gene orf256" Biomolecules, vol. 2, pp. 228-239, 2012.

[5] A.K. Noyszewski, "Mitochondrial sequence diversity among alloplasmic and euplasmic triticum species" Doctoral dissertation, North Dakota State University, Fargo, 2013.

[6] B. Wei, R. Jing, C. Wang, J. Chen, X. Mao, X. Chang, J. Jia, "Dreb1 genes in wheat (Triticum aestivum L.): development of functional markers and gene mapping based on SNPs" Mol Breeding, vol. 23, pp. 13-22, 2009.

[7] S. Lucas, E. Durmaz, B.A. Akpınar, "The drought response displayed by a DRE-binding protein from Triticum dicoccoides" Plant Physiol. and Biochem., 49: 346e351, 2011.

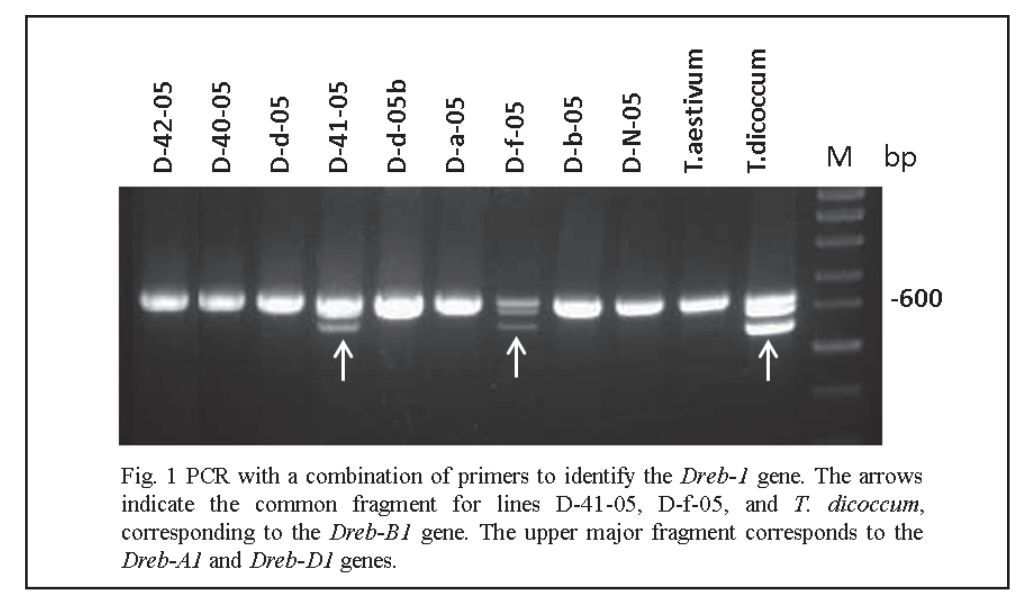

